\title{
Frequency Capture Characteristics of Gearbox Bidirectional Rotary Vibration System
}

\author{
Ruqiang Mou, Li Hou, Zhijun Sun, Yongqiao Wei and Bo Li \\ School of Manufacturing Science and Engineering, Sichuan University (610065), Chengdu, China
}

(Received 25 September 2013; accepted 9 June 2014)

\begin{abstract}
According to the characteristics of the gearbox and Lagrange mechanics, in this paper a bidirectional rotary vibration system dynamics model of the gearbox is established, using MATLAB to simulate the model, study the vibration characteristics of the system in both horizontal and vertical directions, and compare it to existing simplified models. Through the analysis of the model, the conditions of the system that produce frequency trapping are studied, and the frequency factors of the system are obtained. The results indicate that reducing eccentric mass, eccentricity, and rotary damping, and increasing damping movement, bearing stiffness, and input torque can improve system response speed and reduce the amplitude, which can avoid frequency trapping of the system. The study provides a theoretical basis for optimization and installation of the gearbox system.
\end{abstract}

\section{INTRODUCTION}

Due to the rapid development of modern industry, gears have become one of the key parts in the modern industry. The gear box has a fixed gear ratio, transmission torque, compact structure, etc., which is now widely used in various machines. But the gearbox forces are complex. Elastic and inertial forces needed to withstand the complex alternating load. The frequency of trapping will happen, allowing the system deformation or vibration, which affects the normal operation of the machine, or even damages it.

Frequency capture is a special nonlinear vibration phenomenon. During this phenomenon, when the external excitation frequency is close to the natural frequency of the system, the external excitation frequency will synchronize with the system's natural frequency, the frequency trapping. ${ }^{5}$ It has been established that a class of non- ideal vertical vibration system vibration models, are being used to study the movement and rotary damping effect on the frequency capture, but it did not analyse other parameters on the frequency trapping effects. ${ }^{1}$ A reverse rotation dual-drive vibration system model has been created to study the vibration frequency of the system model that achieves trapping conditions. ${ }^{2}$ A vertical direction vibration model has been simplified to a general autonomy system, and the frequency of trapping phenomenon has been included, but it is not specific to the analysis of the physical parameters. ${ }^{3}$ Research $^{4}$ on frequency capture simulation established the elastic support rotor system of the digital prototype model to study the torque and spring stiffness on the frequency of trapping effects. Researchers have studied the wind turbine blade fatigue loading frequency trapping. ${ }^{5}$ Other research ${ }^{6}$ studied the engineering nonlinear vibration problem, empha- sizing the need to effectively take advantage of favourable nonlinear vibration and control of harmful nonlinear vibration.

This selection of a particular model of a gear transmission gearbox as the research object, which is based on the existing research, ${ }^{7-14}$ regards the gearbox system as a nonlinear vibration system, takes into account the horizontal and vertical vibration of the gearbox, studies the frequency trapping conditions in the course of gearbox work, analyses the system parameters on frequency capture by a numerical simulation method, and achieves a frequency trapping gearbox digitization and visualization, which provides a theoretical basis for the dynamic optimization of the gearbox system.

\section{MATHEMATICAL MODEL OF GEARBOX BIDIRECTIONAL ROTARY VIBRATION SYSTEM}

A gearbox is mainly composed of the input shaft, output shaft, driving wheel, driven wheel, the motor (power source), the lid, and the support member. The gearbox is operated by driving the motor under the effect of the input operation, which leads to the driven wheel running. In the gearbox bidirectional rotary vibration system model shown in Fig. 1, m1 and m2, respectively, represent the driving wheel and the driven wheel produced by the eccentricity. When the motor rotates, the eccentric block generates two driven body vibration exciting forces. The vibration body from the horizontal, vertical, and torsional vibration in the direction, taking into account the torsional vibration little effect on the system, ${ }^{2}$ thus ignoring the reverse direction vibrations.

In the simplified model, the body motion coordinates $x$, $y$, and two eccentric rotations of the rotor phase indicate the 


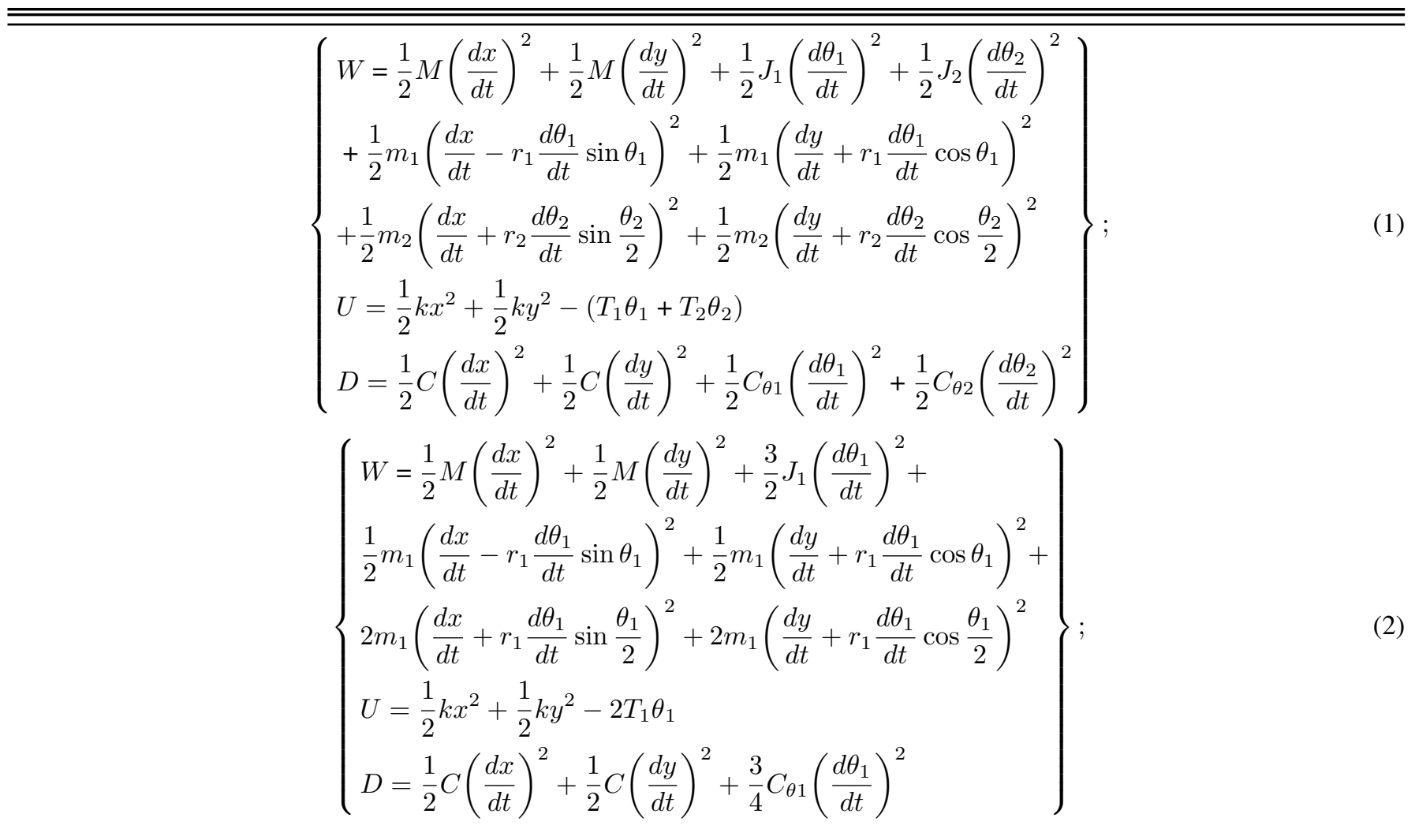

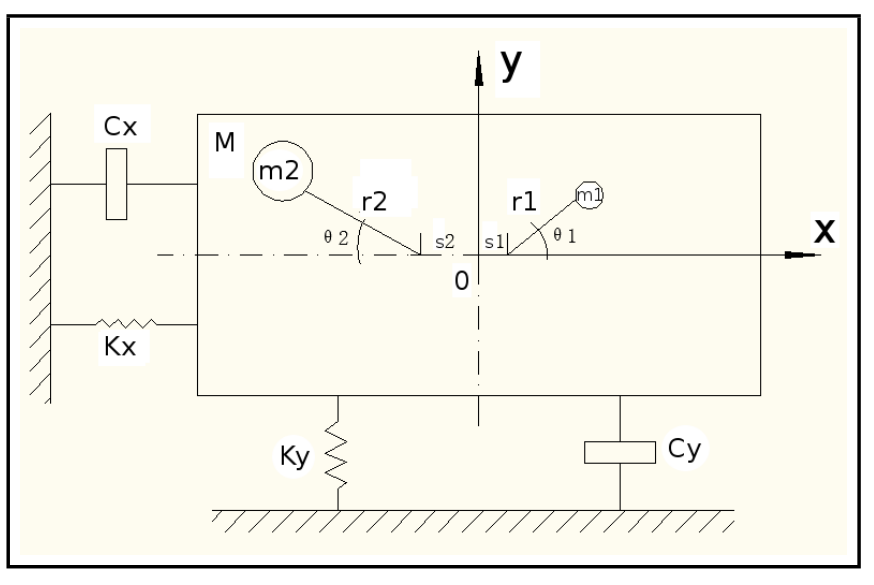

Figure 1. Integrated vibration model of the gearbox system.

generalized coordinates. According to the principle Lagrange equations of motion used to obtain the mathematical model, this is expressed as:

The system is a gear train. Using the system transmission ratio $i=2$, according to the transmission principle, the following relation can be deduced: $r_{2}=2 r_{1} ; \theta_{2}=2 \theta_{1} ; C_{\theta 2}=2 C_{\text {theta } 1}$; $m_{2}=4 m_{1} ; T_{2}=2 T_{1} ; J_{2}=8 J_{1}$.

Taking the above relationship into Eq. (1), the Eq. (2) is obtained, where: $W$ is the vibration system kinetic energy; $U$ is the system potential energy; $D$ is the energy dissipation function; $x, y, \theta_{1}, \theta_{2}$ are the vibration substrate horizontal displacement and the vertical displacement; eccentricity blocks 1 and 2 of the rotation angle; $M, m_{1}, m_{2}, r_{1}, J_{1}, J_{2}$ represent the vibration of the substrate mass, the drive gear, and driven gear eccentric mass, inertia quantitative and qualitative heart eccentricity; $k, C, C_{1}, C_{2}$ are the supporting stiffness coefficients, damping, and two rotary movement damping; and $T$ is the input torque of the motor. By the Lagrange equation

$$
\frac{\mathrm{d}}{\mathrm{dt}}\left(\frac{\partial W}{\partial \dot{q}_{i}}\right)-\frac{\partial W}{\partial q_{i}}+\frac{\partial U}{\partial q_{i}}+\frac{\partial D}{\partial \dot{q}_{i}}=Q_{i}(t) ;
$$

the differential equations (4) and (5) can be obtained (see the top of the next page), where $m=m_{1} ; r=r_{1} ; J=J_{1} ; \theta=\theta_{1}$; $T=T_{1} ; C_{1}=C_{\theta 1}$.

\section{RESPONSE CHARACTERISTICS OF THE VIBRATION SYSTEM FREQUENCY TRAPPING}

According to the analysis, the following initial conditions were obtained: $r=4 m, M=60000 \mathrm{~kg}, m=800 \mathrm{~kg}$, $k=2600 \mathrm{~N} / \mathrm{m}, J=28000 \mathrm{kgm}^{2}, c=62000 \mathrm{Ns} / \mathrm{m}$, $c_{1}=901.8 \mathrm{Ns} / \mathrm{m}, T=1406.46 \mathrm{Nm}$.

Taking these conditions into Eq. (3), the numerical simulation software MATLAB was used to analyse the system, obtain the system horizontal and vertical displacement, and velocity and displacement response spectrum shown in Fig. 2.

As can be seen in Figs. 2(b)-(e), the horizontal and vertical directions of the vibration characteristics are similar, and the amplitude is approximately $5 \mathrm{~mm}$. The difference lies in the amplitude of the horizontal symmetry about the $x$ axis and the vertical symmetry slightly to the negative direction of the $y$-axis. According to the analysis, the two eccentric masses, eccentricity, rotating speed, and the direction of rotation are different. When the two eccentric masses rotate, the two eccentric blocks moves the system in the horizontal direction, 


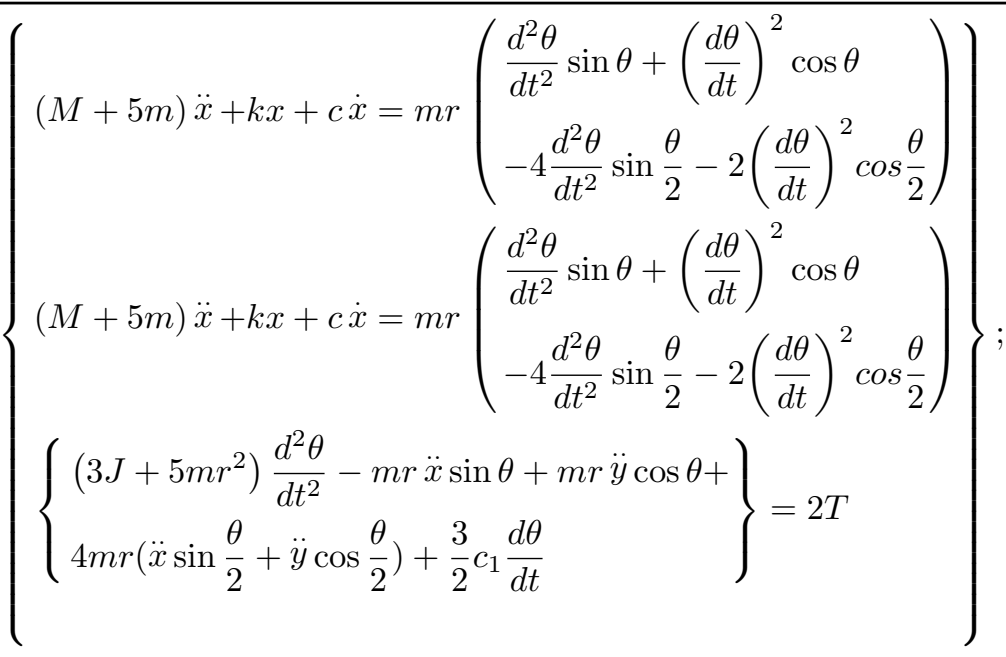

$$
\begin{aligned}
& \left\{\begin{array}{l}
(M+5 m) \ddot{y}+k y+c \dot{y}=m r\left(\begin{array}{l}
\left(\frac{d \theta}{d t}\right)^{2} \sin \theta-\frac{d^{2} \theta}{d t^{2}} \cos \theta+ \\
2\left(\frac{d \theta}{d t}\right)^{2} \sin \frac{\theta}{2}-4 \frac{d^{2} \theta}{d t^{2}} \cos \frac{\theta}{2}
\end{array}\right) \\
\left(\begin{array}{l}
\left(3 J+5 m r^{2}\right) \frac{d^{2} \theta}{d t^{2}}+m r \ddot{y} \cos \theta+ \\
4 m r \ddot{y} \cos \frac{\theta}{2}+\frac{3}{2} C_{1} \frac{d \theta}{d t}
\end{array}\right)=2 T
\end{array}\right\} ;
\end{aligned}
$$

respectively. One by one by shear displacement, horizontal displacement occurs on the final $x$-axis symmetry. However, when the two eccentric masses rotate in two eccentric vertical directions, respectively, the system increases with the displacement and with reduction; but due to the presence of two eccentric phases, this causes the vertical displacement not to remain symmetric about the $x$-axis, and moves the symmetry slightly in the negative direction on the $y$-axis.

From Figs. 2(c)-(e), it can be seen that the system vibration frequency is $0.122 \mathrm{~Hz}$, which was captured by the system with the natural frequency $0.12 \mathrm{~Hz}$. From Figs. 2(a)-(d) it can be seen that the input shaft speed is about $1.52 \mathrm{rad} / \mathrm{s}$ less than the rated speed, which indicates that, under the given parameters, the mechanical system will affect the rotation of the input shaft.

If only the vertical vibration is considered, then (3) can be simplified as in Eq. (5). ${ }^{3}$

The initial conditions remain constant, and by using MaATLAB's numerical simulation, the system vertical displacement, velocity, and displacement response spectrum were obtained, as shown in Fig. 3.

From Fig. 3 and 4, we can find that the system vertical vibration characteristics are similar in both the integrated model and the simplified model, and the frequency trapping phenomena have occurred, resulting in the input shaft speed being less than the rated speed, affecting the normal operation of the whole system. But the former model takes into account the horizontal and vertical vibrations in both directions, which is more in line with the actual working conditions.

If the parameters are taken as $M=60000 \mathrm{~kg}, m=800 \mathrm{~kg}$, $r=4 \mathrm{~m}, J=28000 \mathrm{kgm}^{2}, k=2600 \mathrm{~N} / \mathrm{m}, c=62000 \mathrm{Ns} / \mathrm{m}$, $c_{1}=901800 \mathrm{Ns} / \mathrm{m}, T=1406460 \mathrm{Nm}$, the vibration characteristic curve before the system is simplified can be obtained, as shown in Fig. 4, and the simplified system vibration characteristic curve can be obtained, as shown in Fig. 5.

Comparing Figs. 4 and 5, it can be seen that the frequencies of the systems are not trapping, and the input shaft has a stable speed in $2.21 \mathrm{rad} / \mathrm{s}$, which has reached the rated speed. In the non-occurrence frequency capture, through the two model system response and frequency spectrum comparison, it was found that the former is more responsive than the latter. The former taking $20 \mathrm{~s}$ to stabilize the vertical vibration, while the latter requires $100 \mathrm{~s}$ to become stable. The former amplitude of the horizontal and vertical direction is not more than $15 \mathrm{~mm}$, and the latter peak vibration is large, close to $30 \mathrm{~mm}$, which can seriously devastate the supporting member. Thus, increasing the horizontal spring damping device helps to quickly reduce vibration.

\section{EFFECT OF SYSTEM PARAMETERS ON THE VIBRATION SYSTEM FREQUENCY CAPTURE}

From Eq. (3), it can be seen the system parameters such as eccentric mass, eccentricity, input torque, bearing stiffness, damping, and rotary movement damping will affect the vibrations of the gear box system. Therefore, it is important to anal- 


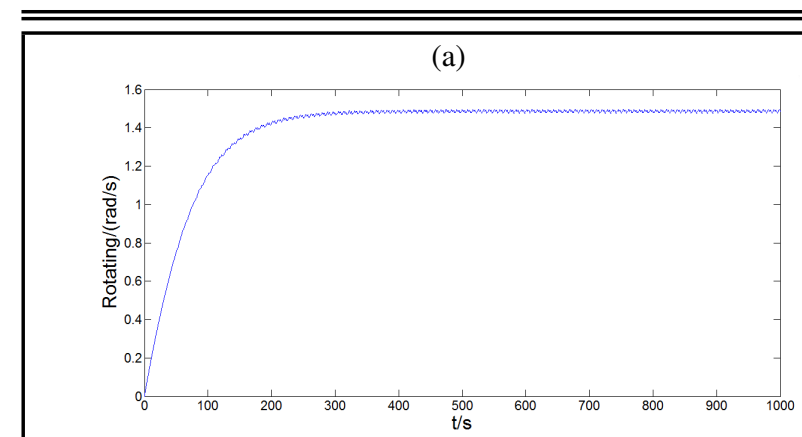

(b)

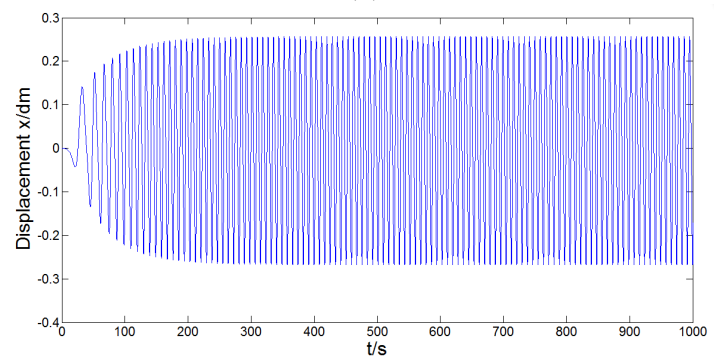

(c)

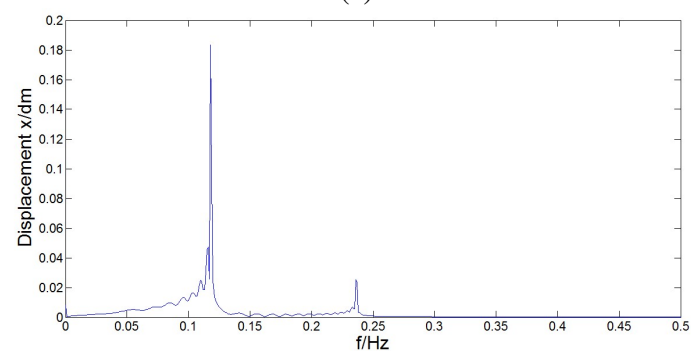

(d)

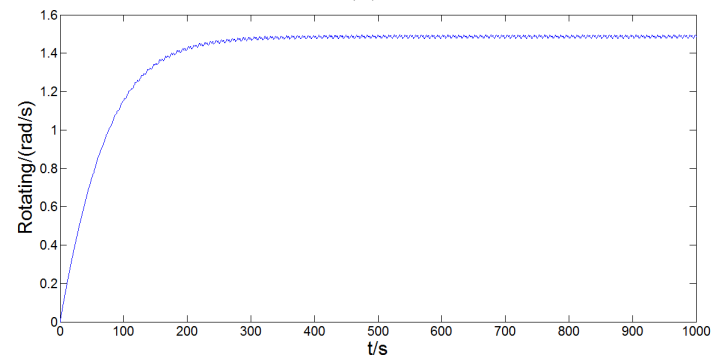

(e)

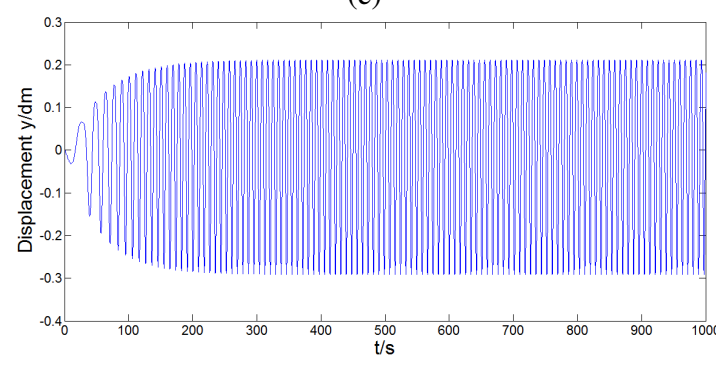

(f)

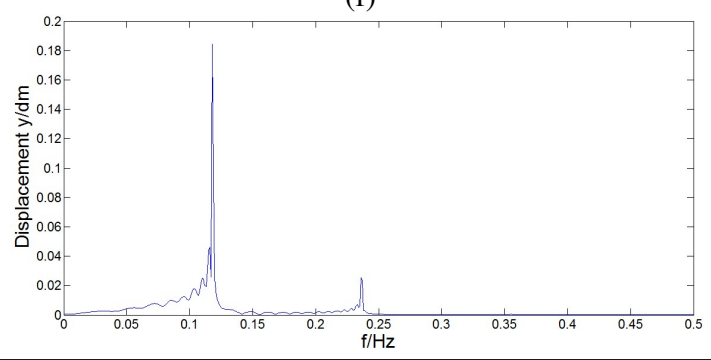

Figure 2. System horizontal and vertical displacement, velocity, and displacement response spectrum. (a)

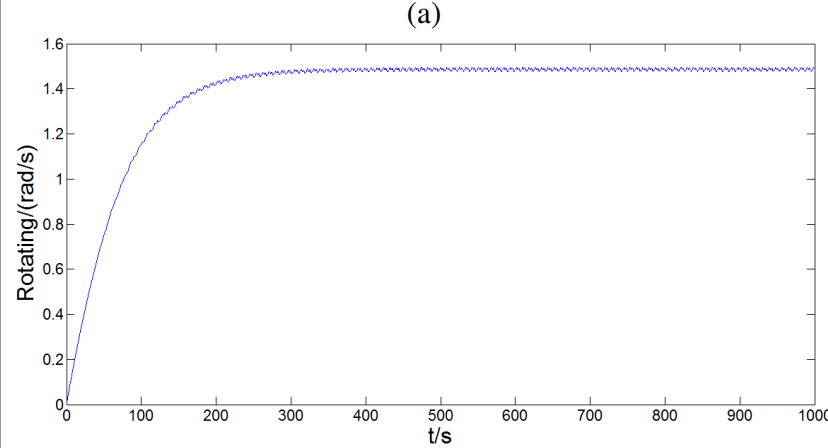

(b)

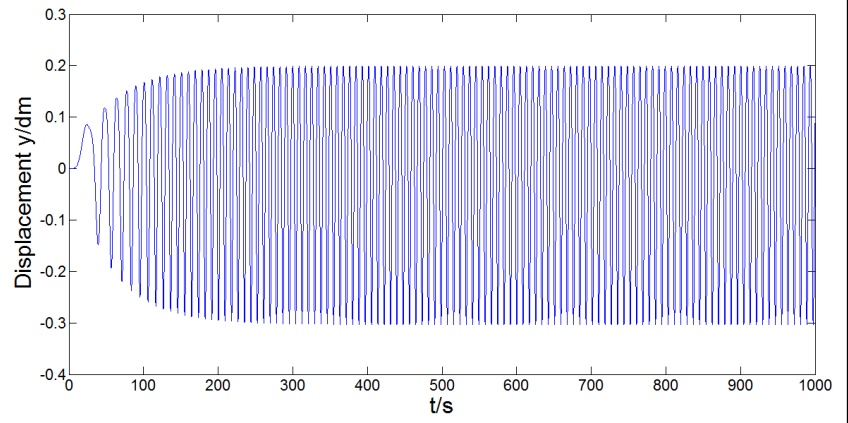

(c)

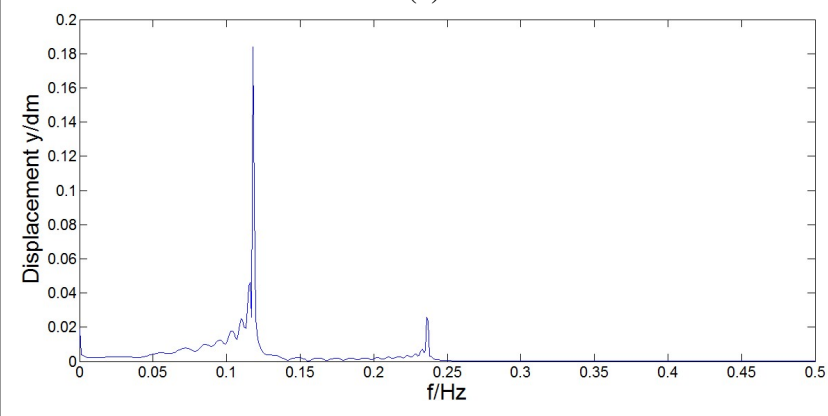

Figure 3. System vertical displacement, velocity, and displacement response spectrum.

yse the system parameters, which obtain the effect of the system parameters on the system trapping phenomenon.

The initial parameters are $M=60000 \mathrm{~kg}, m=800 \mathrm{~kg}$, $r=4 \mathrm{~m}, J=28000 \mathrm{kgm}^{2}, k=2600 \mathrm{~N} / \mathrm{m}, c=62000 \mathrm{Ns} / \mathrm{m}$, $c_{1}=901.8 \mathrm{Ns} / \mathrm{m}, T=1406.46 \mathrm{Nm}$.

Using the numerical simulation software MATLAB for Eq. (4), the system steady speed of the input shaft effect curve was obtained. In the case of the other parameters' constants, the eccentric mass effect on the input rotation speed is shown in Fig. 6.

Figure 6 shows the influence curve of the eccentric mass on the input speed, from which it can be seen that, as the eccentric mass $m$ increases, the system response slows, and the stable speed of the input shaft decreases, the frequency of trapping system, meaning the system cannot reach the rated speed. While the vibration characteristics of the system diagram analysis found that the eccentric mass system will inevitably lead to an increase in amplitude, and that eccentric mass has a greater impact on the vibration system, it should be noted that during 


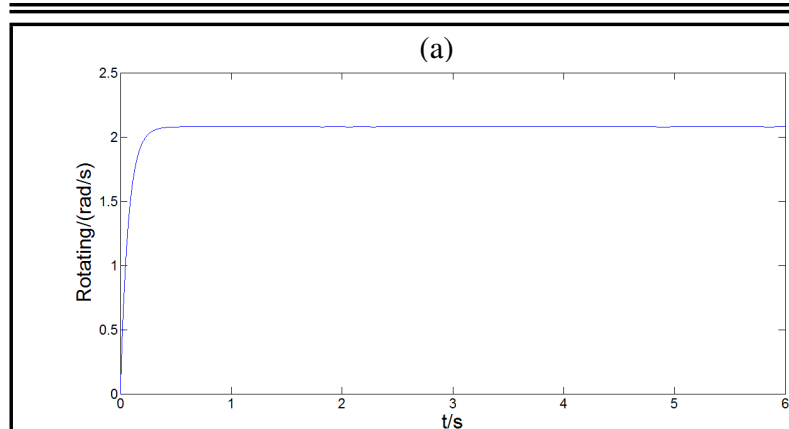

(b)

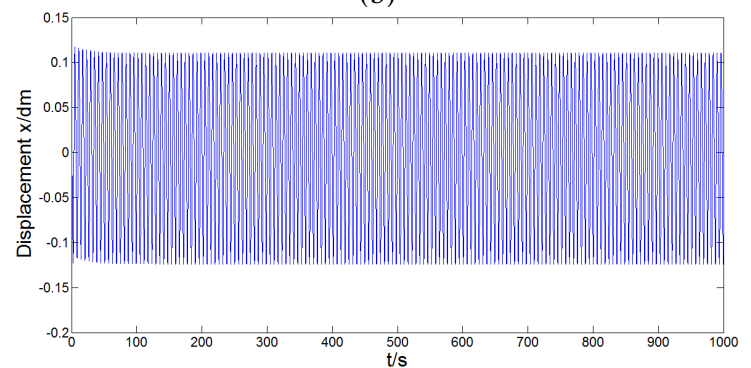

(c)

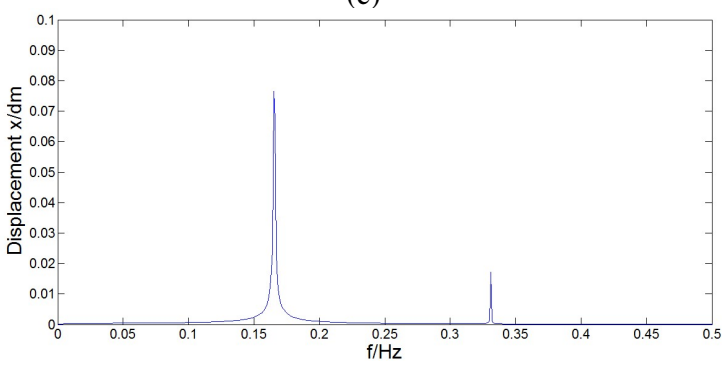

(d)

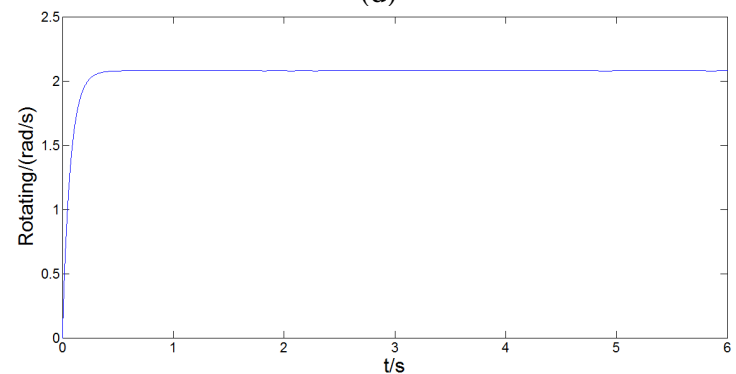

(e)

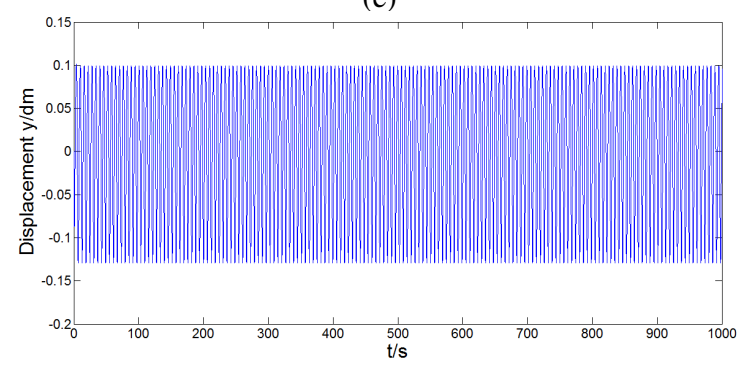

(f)

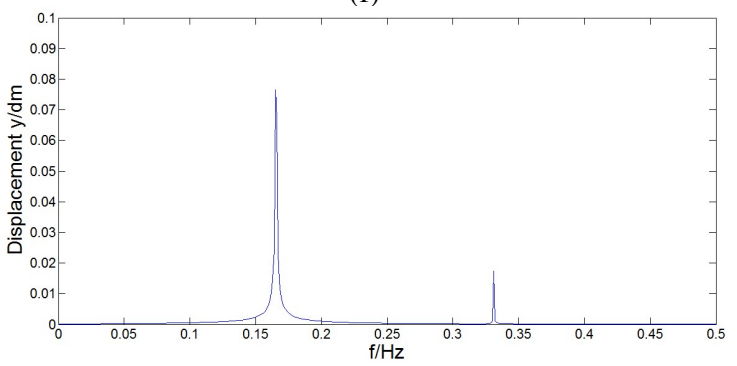

Figure 4. Simplified former system vibration characteristics curve. (a)

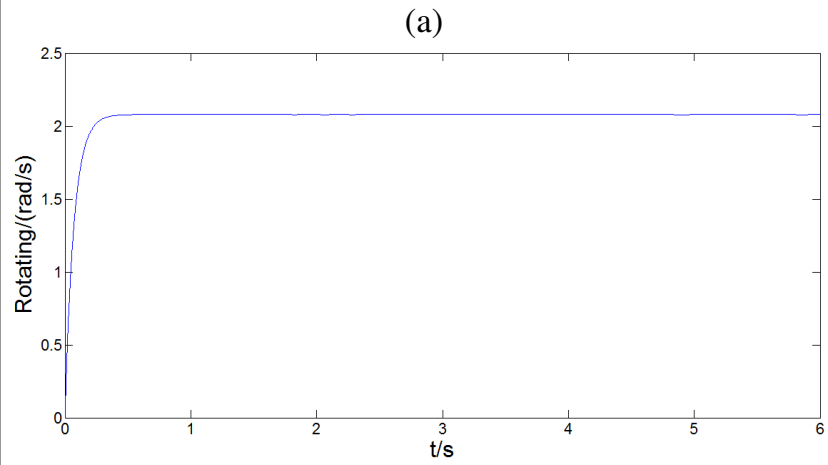

(b)

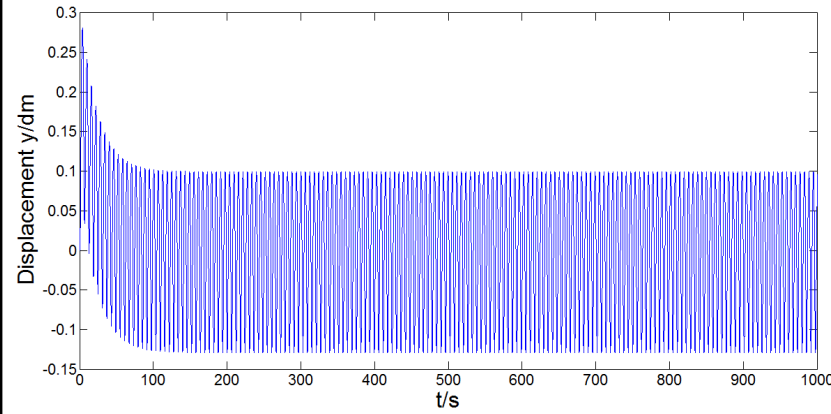

(c)

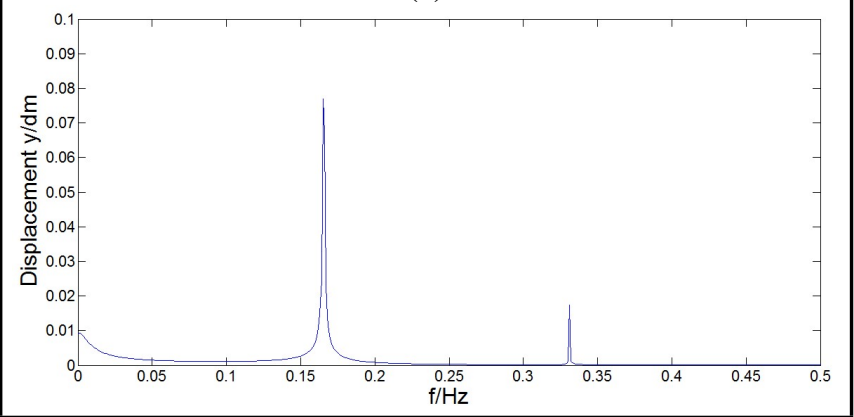

Figure 5. Simplified system vibration characteristic curve.

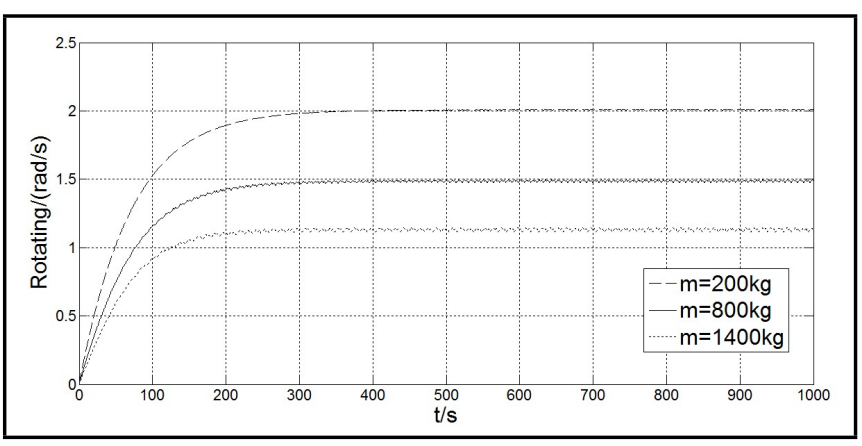

Figure 6. Eccentric mass effect on the input speed.

the design of the gearbox system, the eccentric mass must be strictly controlled.

Figure 7 show the movement damping effect on the input rotational speed curve, from which it can be seen that, as the mobile damping increases, the input shaft speed first decreases and then increases, so moving the damping can effectively avoid the natural frequency, which helps to avoid the trapping frequency. Depending on the system material properties, a generally larger movement damping factor, such as 


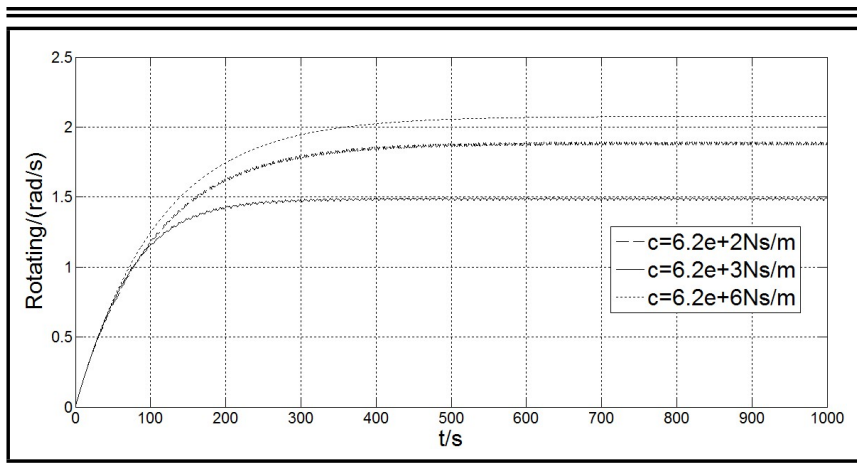

Figure 7. Mobile damping effect on the input speed.

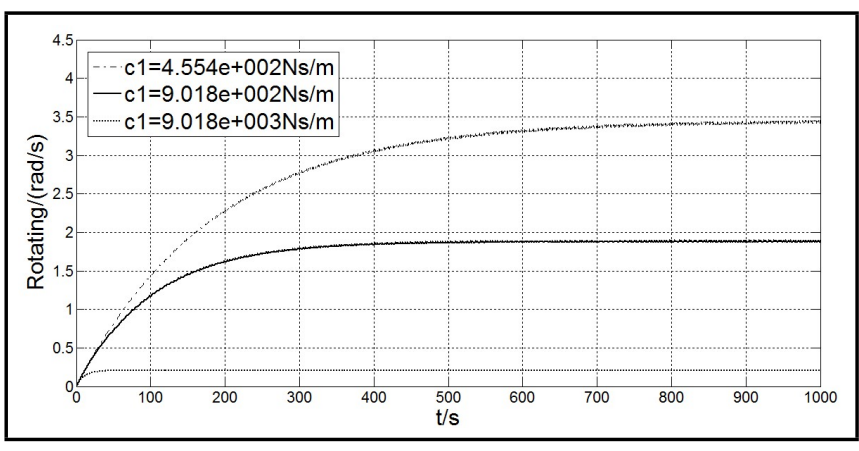

Figure 8. Rotary damping effect on the input speed.

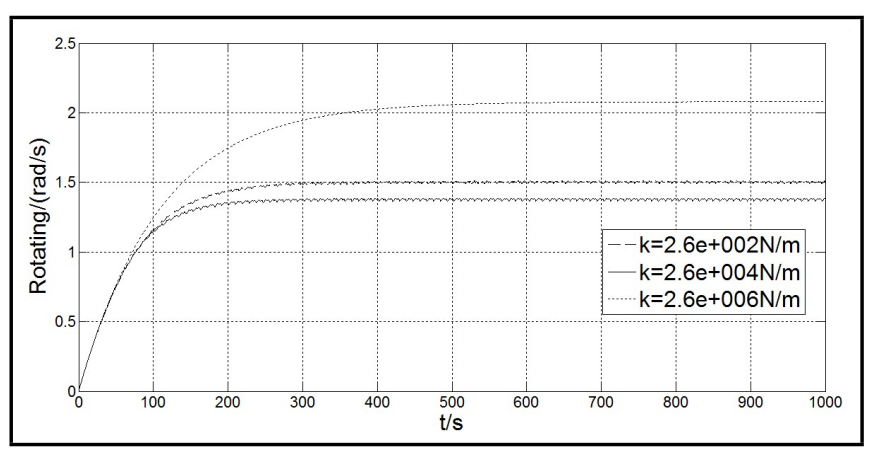

Figure 9. Bearing stiffness effect on the input speed.

$c=6.5 \times 10^{6}$, can effectively reduce the amplitude, and avoid the frequency trapping conditions of the system, to ensure that the system works normally.

Figure 8 shows a rotary damping effect on the input rotational speed curve. From Fig. 8 it can be seen that, as the rotation damping increases, and the smaller the amplitude of the system, the input shaft speed decreases, resulting in the input shaft speed being less than the rated speed. Therefore, in order to avoid the system experiencing frequency trapping, ensure the amplitude of the system in the allowable range, and reduce the rotational damping as far as possible.

Figure 9 shows the bearing stiffness on the impact of input speed curve. As the bearing stiffness increases, the system response speed and input shaft speed first decreases, and then increases. When the stiffness is very low, the frequency of the system is easy to capture. When the stiffness is high, the natural frequency of the system has no influence on the frequency trapping. Therefore, in the design of the gearbox system, it

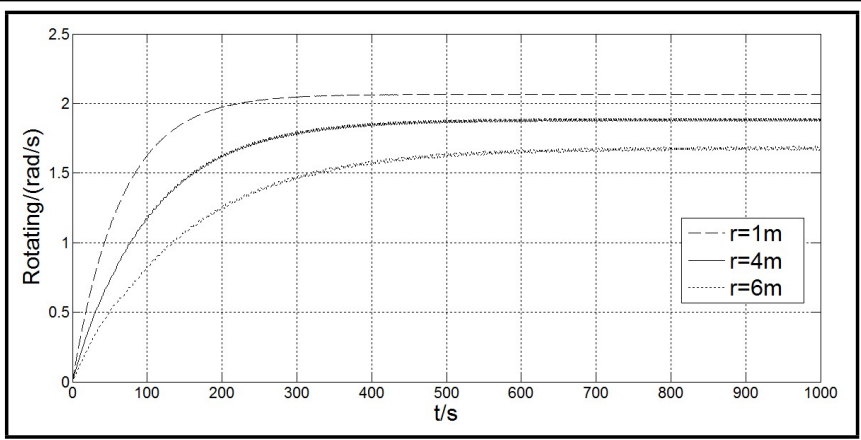

Figure 10. Eccentricity effect on the input speed.

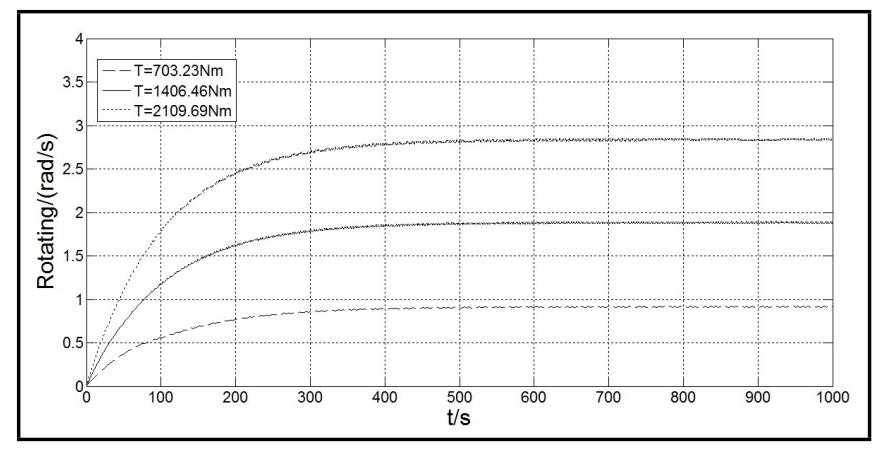

Figure 11. Input torque effect on the input speed.

is best to choose a larger bearing stiffness that can effectively avoid the system frequency trapping.

Figure 10 shows the eccentricity of the input rotational speed curve. It can be seen from the figure that as the eccentricity increases, the system response slows, the input shaft speed decreases, and the system experience frequency trapping, eventually causing the input shaft speed to be less than the rated speed. When the eccentricity is less than $1 \mathrm{~m}$, the input shaft can reach the rated speed, and the system does not experience frequency capturing.

Figure 11 shows the input torque effect on the input speed curve, from which it can be seen that as the input torque increases, the system response speed becomes faster, the input shaft speed increases, the system does not experience frequency trapping, and the system can quickly reach the rated speed. Therefore, improving the system's input torque can effectively avoid the occurrence of frequency capturing.

\section{CONCLUSION}

According to the characteristics of the gearbox, a horizontally- and vertically- integrated dual rotary vibration model of the gearbox system was established, and the model frequency trapping was analysed. The simplified model exists only in the vertical direction vibration. By comparison, the integrated model is more realistic. In addition, this paper, using numerical simulation, analysed the system parameters' (such as the eccentric mass, eccentricity, input torque, bearing stiffness, damping, and rotary movement damping) effects on the 
system frequency capture. In summation, this study accomplishes and discusses the following:

1. According to the characteristics of the gearbox, establishes an integrated vibration model and recreates the system frequency trapping by numerical simulation.

2. When the system does not capture frequency compared with the simplified model, the integrated model has small amplitude and fast response speed; once the system frequency trapping is measured, the integrated vibration model can reflect the characteristics of both horizontal and vertical vibrations in both directions, which can more accurately describe the vibration characteristics of the system.

3. System parameters analysis showed that, for the integrated vibration model, reducing eccentric mass, eccentricity, and rotary damping, and increasing damping movement, bearing stiffness, and input torque can improve the response speed of the system. Also reducing the amplitude and preventing system frequency trapping, and reducing the vibration impact on the system, help the system to quickly reach the rated speed, which helps to ensure the normal operation of the system.

\section{REFERENCES}

1 Xiong, W., Lu, M., and Wen, B. Characteristics of rotating frequency catching of non- ideal vibration systems, $J$. Hunan U.: Nat. Sci., 30 (3), 44-48, (2003).

2 Wang, D.-G., Zhao, C.-Y., Ren, Z.-H., and Wen, B.-C. Frequency-based capture control on revertible dual-motordriven vibration system, Chinese J. Constr. Mach., 6 (3), 282-286, (2008).

3 Zhang, Nan, et al. Characteristics of frequency capture of nonlinear vibration systems, J. Dongbei U.: Nat. Sci., 30 (8), 1170-1173, (2009).

4 Han, Qingkai, et al. Frequency capture simulation and experiment of a rotor system with elastic supports, J. Vib. Shock, 27 (8), 63-66, (2008).
5 Zhang, L. and Wu, J. Frequency capture characteristics in wind blade fatigue loading process, J. Sichuan U.: Eng. Sci. Ed., 43 (6), 248-252, (2011).

6 Wen, Bangchun, et al. Engineering nonlinear vibration, Science Press, Beijing, 2008.

7 Qingkai, H., Wang, L., Yao, H., and Wen, B. Simulation and experiment analyses on resonance for a rotor system with elastic supports, Proc. IDETC/CIE 2007, Las Vegas, Nevada, USA, (2007). http://dx.doi.org/10.1115/detc200734420

8 Zhu, Caichao, et al. Research of nonlinear dynamic characteristics of wind power gear box system, J. Mech. Eng., 41 (8), 203-206, (2005).

9 Xiong, Wanli, et al. Mechanism of electromechanicalcoupling on self-synchronous vibration and vibratory synchronization transmission, J. Vib. Eng., 13 (3), 325-331, (2000).

10 Chunyu, Z., Degang, W., Hao, Z., Jie, L., and Bangchun, W. Frequency capture of vibration system with two-motor drives rotating in same direction, Chinese J. Appl. Mech., 26 (2), 283-287, (2009).

11 Liu, Z., Zhou, X., Ye, H., Xiang, W., and Yang, S. Design and realization of system software for vibration sources signal separation of gearbox, Mach. Tool Hydr., 36 (5), 198201, (2008).

12 Liu, J., Yang, J., Yang, M., Zeng, J., and Yang, J. Vibration signal acquisition and analysis system of wind turbine gearbox based on MATLAB and VC, Guangdong Elec. Power, 26 (6), 70-74, (2013).

13 Shen, Guoji, et al. The estimates of gearbox vibration signal phase based on Wigner distribution, Chinese J. Mech. Eng., 40 (9), 186-188, (2004).

$14 \mathrm{Li}$, Hao, et al. The noise reduction of gearbox vibration signal processing based on Wavelet transform, Mach. Des. Мапи., 3, 82-83, (2013). 